\title{
La construcción de indicadores biográficos mediante el análisis reticular del discurso. Una aproximación al análisis narrativo-biográfico
}

\author{
Joan Miquel Verd, Universidad Autónoma de Barcelona ${ }^{1}$
}

\begin{abstract}
Resumen
El artículo desarrolla una forma novedosa de análisis narrativo-biográfico que se fundamenta en las aplicaciones de la perspectiva de redes sociales a los análisis de textos. Partiendo de la base de que es deseable cierto grado de distanciamiento y objetivación en el procedimiento de análisis de cualquier texto, se revisan en primer lugar aquellas aproximaciones biográficas que se han movido en esta dirección. A continuación se presenta una aproximación formal al análisis narrativo-biográfico que se enmarca en la lógica del análisis reticular del discurso. En el marco de esta aproximación se muestra la posibilidad de identificar el contenido empírico de la mayoría de indicadores biográficos más comúnmente utilizados en la investigación biográfica.
\end{abstract}

Palabras clave: análisis biográfico, indicadores biográficos, análisis narrativo, análisis de redes textuales.

\begin{abstract}
The article develops a new biographic-narrative method based on the application of the social network perspective to text analysis. First of all, biographic methods that take a certain degree of distance and objectification in the process of analysis are reviewed, taking for granted that this attitude is desirable in any kind of research. Next, a formal approach to biographic-narrative analysis based on network discourse analysis is presented. In the framework of this approach, it is possible to identify the empirical content of most of the biographic indicators commonly used in biographic research.
\end{abstract}

Key words: biographic analysis, biographic indicators, narrative analysis, network text analysis.

\footnotetext{
${ }^{1}$ Departamento de Sociología - Centre d'Estudis Sociològics sobre la Vida Quotidiana i el Treball. Enviar correspondencia a: JoanMiquel.Verd@uab.es
} 


\section{Introducción}

A partir de la década de los noventa han proliferado en el conjunto de las ciencias sociales las investigaciones denominadas "narrativas". Esta eclosión ha llevado a que la investigación narrativa sea cualificada en ciertos manuales como un nuevo estilo o método de investigación (Bell, 2002/1999; Fielding y Lee, 1998). En la práctica, los procedimientos de análisis que se engloban bajo este término son múltiples, así como las filiaciones teóricas y epistemológicas de sus autores/as. EI interés común es "cómo una información extraída de la narración de una historia se puede estructurar de forma que produzca unas conclusiones válidas para la investigación" (Bell, 2002/1999: 28). De hecho, casi podría afirmarse que el único punto de contacto de todas estas investigaciones es la producción (habitualmente a través de entrevistas semiestructuradas o no estructuradas) de datos cuya forma lingüística es el relato.

De todos modos, si el uso de relatos como material empírico fuese el único distintivo de la investigación narrativa resultaría absolutamente injustificado afirmar que la investigación narrativa es "nueva". Los relatos, y en particular los de carácter biográfico, vienen utilizándose en la investigación social cualitativa desde sus inicios.

No obstante, sí pueden considerarse como novedosos los análisis narrativos. En relación con la investigación narrativa -que podría definirse en términos globales como el método que se fundamenta en la recolección/producción de datos en forma de relato y en su posterior interpretación y análisis mediante procedimientos adaptados- el análisis narrativo hace referencia más concretamente a las etapas de presentación y reducción de los datos y a la obtención y verificación de conclusiones, que caracterizan la fase analítica de una investigación cualitativa (Miles y Huberman, 1994). Es en esta fase de análisis, más que en la de construcción de los datos, en la que últimamente se están produciendo desarrollos novedosos. 
Buena parte de estos desarrollos novedosos se caracterizan por desarrollar algún tipo de procedimiento que permita la objetivación por parte del analista del material textual analizado. Dicha objetivación suele conseguirse mediante un cierto grado de formalización del análisis, aspecto en el que profundizaremos más adelante. Esta tendencia no es ajena a los cambios que desde los años ochenta se están dando en esta misma dirección en el conjunto de los análisis cualitativos de textos.

En el marco de estas nuevas aproximaciones al análisis narrativo el artículo muestra cómo los análisis de textos que utilizan la formalización reticular pueden ser provechosamente utilizados en el análisis de narraciones biográficas (también conocidas como relatos de vida o biográficos) y más concretamente en la construcción de lo que he llamado indicadores biográficos.

Con este propósito, el artículo se ha estructurado en dos partes principales. En la primera de estas dos partes, formada por los epígrafes 1 y 2 , se realiza una primera aproximación al tipo de estructuras narrativas presentes en cualquier relato biográfico y se revisan las aproximaciones que desde diferentes posicionamientos se han planteado su análisis. Posiblemente no se ha conseguido una exhaustividad absoluta en cuanto a las obras referenciadas, aunque tampoco es este el objetivo, sino más bien revisar aquellos estilos de análisis narrativo que han avanzado en la dirección de introducir cierta formalización en el proceso de tratamiento de los datos. Por lo tanto quedan fuera del artículo innumerables autores que habiendo trabajado en el campo del análisis narrativo-biográfico, no han tenido como preocupación central el análisis de las estructuras narrativas del relato.

En la segunda parte, formada por los epígrafes 3 y 4 , se presenta un modelo específico de análisis narrativo-biográfico fundamentado en el análisis reticular del discurso y se revisan las principales aportaciones que supone esta aproximación al conjunto de perspectivas en que se inserta. Al tiempo que la aproximación propuesta recoge ciertos elementos ya destacados en modelos anteriores, permite avanzar en el terreno de la identificación empírica de ciertos conceptos ya corrientes en los análisis biográficos. He denominado indicadores biográficos a esta versión empírica de los conceptos citados. Finalmente, el artículo termina con un pequeño epígrafe destinado a las conclusiones, en que se resumen algunas ventajas de la aproximación propuesta, tanto desde el punto de vista metodológico como desde el punto de vista teórico. 


\section{La estructura sincrónica y diacrónica en la narración}

La forma narrativa es una de las formas lingüísticas con un mayor grado de estructuración interna. De hecho, desde los trabajos pioneros de Propp (1977/1968) los lingüistas no han dejado de analizar las características estructurales de este tipo de discursos. En este sentido, desde finales de los años sesenta y durante toda la década de los setenta la narratología se ha impuesto como una nueva forma de contemplar una historia o un relato (verbal o escrito), en la que "la exégesis ha quedado sustituida por un enfoque sistemático que identifica las estructuras narrativas fundamentales" (Larsen, 1993: 157). Este modo de aproximarse a los relatos ha producido también destacados trabajos en el campo de la sociología, de los cuales posiblemente los más conocidos sean los llevados a cabo por Labov y sus colaboradores (Labov y Waletzky, 1967; Labov, 2000/1972; para una evaluación retrospectiva veánse Labov, 1997, y Schegloff, 1997).

Este elevado grado de estructuración fundamenta en buena medida la "nueva oleada" de análisis narrativos mencionada en la introducción, puesto que permite el desarrollo de aproximaciones de carácter formalizado. En este contexto, entiendo como formalizado un análisis que vaya más allá de una simple enumeración temática $\mathrm{y} / \mathrm{u}$ ordenada de los acontecimientos narrados en el relato, en que se haga uso de expresiones simbólicas propias de lenguajes algebraicos o vinculadas a esquemas $u$ otros dispositivos gráficos. En todos estos casos, el rédito de la formalización es múltiple: permite reducir el volumen de información que se analiza - lo cual redunda a su vez en unas mayores posibilidades analíticas y comparativas-, hace transparente el proceso y reduce la ambigüedad de los conceptos, y facilita la validación recíproca entre la abstracción del concepto y su dimensión operativa atribuida en el texto biográfico. ${ }^{2}$

\footnotetext{
${ }^{2}$ Dicha formalización no debería suponer el olvido del análisis contextual del texto y de sus condiciones de producción, así como tampoco de sus aspectos lingüísticos y pragmáticos.
} 
Debe señalarse que el tipo de análisis de que venimos hablando puede identificarse con la segunda de las dos grandes orientaciones en que Van Dijk (1999/1998) divide los análisis del discurso. Según este autor es posible distinguir entre análisis estratégicos (que abordan el análisis del discurso como proceso) y análisis estructurales (que abordan el análisis del discurso como producto). Además, dentro de este segundo tipo de análisis es posible distinguir entre análisis interesados por la estructura sincrónica y análisis interesados por la estructura diacrónica. A partir de estas distinciones se ha elaborado el cuadro 1.

\begin{tabular}{|c||c|c|}
\hline \multicolumn{1}{|c||}{ Análisis estratégicos } & \multicolumn{2}{c|}{$\begin{array}{c}\text { Análisis estructurales } \\
\text { (el discurso como producto) }\end{array}$} \\
\hline $\begin{array}{c}\text { Atención a la dinámica y al proceso en la } \\
\text { interacción discursiva. El discurso se entiende } \\
\text { como resultado de las estrategias discursivas } \\
\text { utilizadas en la interacción }\end{array}$ & $\begin{array}{c}\text { Atención a las categorías, } \\
\text { clasificaciones y } \\
\text { estructuras conceptuales } \\
\text { básicas de carácter } \\
\text { estático }\end{array}$ & $\begin{array}{c}\text { Atención a la estructura } \\
\text { ordenada secuencialmente } \\
\text { en términos temporales, } \\
\text { causales o en base a otros } \\
\text { tipos de encadenamiento }\end{array}$ \\
\hline
\end{tabular}

Cuadro 1. Orientaciones principales en el análisis del discurso. Fuente: elaboración propia a partir de van Dijk (1999/1998: 77) y Martí (2000: 76).

Los análisis narrativos se desarrollan tanto en torno a la estructura sincrónica como en torno a la estructura diacrónica, aunque es esta segunda la que en comparación con otras formas lingüísticas se ajusta en mayor medida a una estructura canónica que suele presentar escasas variaciones (Adam, 1992; Calsamiglia y Tusón, 1999; Renkema, 1999/1993). Resulta interesante relacionar estas dos estructuras con la distinción ya clásica en las ciencias cognitivas entre memoria (o conocimiento) semántica y memoria (o conocimiento) episódica. La memoria semántica hace referencia a las estructuras conceptuales básicas que nos permiten aprehender la realidad que nos rodea, se trata en la práctica de las categorizaciones y conocimientos que dan sentido a la realidad que nos rodea. Este tipo de memoria estaría, por lo tanto, en la base de la estructura sincrónica del relato. Por otro lado, la memoria episódica hace referencia al conocimiento vinculado a experiencias vividas en el pasado, que no sólo es posible recordar -cómo podemos hacer con el nombre o profesión de una persona- sino también "revivir". Esta posibilidad de revivir mentalmente un momento previo en el pasado es utilizado por ciertos autores como elemento discriminante entre la memoria semántica y la episódica: en la primera no se recuerdan los hechos que han dado lugar al conocimiento, en la segunda sí (Wheeler, 2000). Esta memoria episódica estaría en la base de la estructura diacrónica del relato. 
Dados los vínculos entre tipos de conocimiento y estructuras en la narración no debería sorprender que los primeros intentos de abordar mediante algún tipo de formalización las dos estructuras mencionadas surjan precisamente en el campo de la ciencia cognitiva. En este sentido deben mencionarse los trabajos de Lehnert y Dyer. Lehnert (1981) aborda la formalización de la estructura diacrónica de la narración con el objetivo de identificar las unidades principales que componen la trama de una narración. Una vez identificadas estas unidades, es posible proceder a la realización de resúmenes de la narración simplemente mediante procedimientos formales. Ello permite a la autora tanto comparar los resúmenes obtenidos con este medio con los resúmenes realizados sin ningún tipo de mecanismo formal como también utilizar el procedimiento seguido para resumir de modo automatizado los principales elementos de una narración. Por su parte Dyer (1983) utiliza una aproximación formal a las narraciones mediante el programa BORIS para detectar en qué medida las diferentes situaciones emocionales son representativas de los objetivos de los actores en la narración y del grado de cumplimiento de sus objetivos. El autor comprueba que en el caso de las narraciones unos aspectos y otros están íntimamente ligados, de modo que mediante la actitud emocional de un determinado narrador es posible identificar las estructuras cognitivas que se encuentran en el trasfondo de su narración.

Ya en el campo de la sociología, Abell (1987; 1988) y Heise (1991) han abordado la representación formalizada de las relaciones de causalidad en las narraciones, aunque mediante procedimientos algo diferentes. El análisis de estructuras de acontecimientos (event structure analysis) de Heise (1991), tiene como objetivo hallar la estructura lógica en un texto de carácter narrativo, utilizando el conocimiento de un "experto" conocedor de los acontecimientos. El interés del método estriba en la posibilidad de conseguir una descripción "objetiva", formalizada y "estructurada" del conjunto de acciones descritas. En este método la narración tiene como función fundamental permitir el acceso a "los hechos", siendo posible "rectificarla" si otras fuentes de datos indican que el experto "está equivocado". Por su parte, la metodología de narrativas comparadas (comparative narratives) de Abell (1987) utiliza la narración de los actores para conseguir una descripción formalizada del conjunto de acciones que llevan a una determinada situación. Para el autor, la representación obtenida será, finalmente, el resultado -con mayor o menor influencia de un modelo teórico- del intento del analista social de describir y explicar el desarrollo concreto de una(s) acción(es) o interacción(es) social(es). Para Abell, en esta tarea, las fuentes de información al alcance del analista son todas las posibles: datos provenientes de entrevistas (a los implicados 
directamente en las acciones que se pretenden describir y a los no implicados directamente), datos provenientes de la observación directa, documentos... Según este autor, "es el trabajo del analista combinar [los datos] en una narrativa coherente $y$, en último término, abordar la cuestión de si la narrativa es generalizable o no" (1987: 45).

A pesar de compartir un mismo tipo de material discursivo, estos trabajos pioneros no han tenido excesiva influencia en el campo más específico de los análisis biográficos, que, como se ha apuntado en la introducción, es el campo específico en el cual se inserta la propuesta que se presenta en el artículo. De hecho, los intentos de formalización en este tipo de análisis se han orientado hacia direcciones bastante diferentes, aunque algunos de ellos tienen pequeños puntos de contacto con las aproximaciones que acaban de reseñarse. Estos intentos se resumen en el siguiente epígrafe.

\section{Algunas aproximaciones significativas al análisis narrativo- biográfico}

Un relato biográfico no es más que un tipo particular de narración. Para destacar este hecho Gergen (1996/1994) denomina a este tipo de relatos autonarraciones. De este modo, como tipo particular de narración, en un relato biográfico pueden también identificarse las dos estructuras principales que se han mencionado en el epígrafe anterior: la estructura sincrónica y la estructura diacrónica. No debe sorprender, entonces, que las aproximaciones formalizadas a los relatos biográficos se sustenten en una de las dos estructuras señaladas, o en algunos casos, en ambas. Utilizaré esta distinción para agrupar en los dos epígrafes siguientes un buen número de aproximaciones que considero significativas.

\subsection{Aproximaciones a la estructura sincrónica de la narración}

Dentro de las aproximaciones interesadas por la estructura sincrónica de los relatos biográficos la influencia de los modelos semióticos y estructurales al estilo de Greimas (1971/1966) es evidente. Para Wengraf (2001: 292-295), esta aproximación es especialmente interesante para el análisis de fragmentos narrativos concretos (lo que este autor llama "microanálisis") que se insertarán en un análisis más global de carácter diacrónico. El objetivo es, especialmente cuando este análisis se desarrolla en los niveles más profundos, obtener la estructura de oposiciones y transformaciones que subyace como patrón de la narración. Con 
todo, su aproximación al análisis narrativo-biográfico se fundamenta principalmente en la dimensión diacrónica, por lo que la trataré con mayor detalle más adelante.

En cambio, el trabajo sobre relatos biográficos de inserción que realizan Demazière y Dubar (1997) se sitúa de lleno en el análisis de la dimensión sincrónica de los relatos, utilizando también una aproximación fundamentalmente estructural. Un concepto clave para estos autores es el de trayectoria subjetiva, entendiendo por ello la historia narrada que justifica y a la vez define la propia identidad. En esta interacción entre identidad y acontecimientos biográficos vividos, que son presentados de forma narrativamente "prototípica", juegan un papel importante las propias instituciones educativas y laborales, puesto que "encuadran y anclan socialmente [a los sujetos] por medio de las categorizaciones [que les aplican]" (Demazière y Dubar, 1997: 304). De este modo, los autores asocian las narraciones a cuatro "grandes mundos" (1997: 308) -de los oficios, de los empleos, de las funciones y de la providencia- expresando con estos términos no sólo la lógica identitaria presente en los relatos, sino también el influjo que sobre la propia trayectoria han tenido los contextos e influencias institucionales que se presentan en el relato.

También la aproximación de Riessman (1993) al análisis de relatos biográficos se desarrolla tomando la narración como medio para estudiar la subjetividad y la identidad de las personas. El siguiente párrafo resume bien cual es la orientación que, para esta autora, debe tomar el análisis narrativo-biográfico:

“El objetivo es ver como los/as entrevistados/as imponen orden en el flujo de experiencias para dar sentido a los acontecimientos y acciones de sus vidas. El enfoque metodológico examina la historia del informante y analiza como se ha conjuntado, los recursos lingüísticos y culturales que utiliza y como convence al oyente de su autenticidad. El análisis en los estudios narrativos saca a la luz las formas de contar la experiencia, no sólo los contenidos a los que se refiere el lenguaje. Nos preguntamos por qué se cuenta la historia de ese modo" (1993: 2; con cursivas en el original).

En el trabajo de Riessman son evidentes las influencias de autores como Gergen (1996/1994) o Bruner $(1987 ; 1991)$ que, con una orientación próxima al construccionismo psicosocial, se interesan también por la función social que ejerce la narración como elemento constitutivo y estructurador de la identidad y experiencia humanas. Se trata de hecho, de conocer cómo los sujetos interpretan y dan sentido a los hechos vividos por medio de la construcción de narraciones. De hecho, Bruner (1991: 20-21) extiende su análisis al conjunto de creencias y reglas sociales, preconizando que éstas se organizan según los mismos principios que generalmente se atribuyen a la narración. Otra de la influencias teóricas que es 
posible detectar en la aproximación de Riessman surge de la idea de "teatralismo" (dramatism) presente en los trabajos de Burke (véase Bruner, 1987; Manning y Cullum-Swan, 1994). Según este autor, los recursos narrativos presentes en un relato pueden abordarse como si de una obra teatral se tratara, de modo que términos como acto, escena, agente, agencia (o instrumento) e intención sirven para identificar cómo se construye la narración.

\subsection{Aproximaciones a la estructura diacrónica de la narración}

En los análisis narrativo-biográficos que se interesan por la estructura diacrónica de la narración, los factores causales suelen tomar un lugar central en los análisis. Como ha destacado Ramos, la narración tiene "una interesante propiedad: que sugiere fuertemente una lógica causal" (2001: 71, con cursivas en el original). La causalidad presente en una narración no es necesariamente una causalidad de carácter mecánico, en el sentido en que puede entenderse el funcionamiento de un sistema que indefectiblemente produce unos resultados a partir de unas determinadas premisas, se trata más bien de razones que para el narrador explican un determinado desarrollo de los acontecimientos. Tal como destaca el propio Ramos (2001), las personas, al narrar explican. Esta explicación puede fundamentarse tanto en circunstancias externas al sujeto como en objetivos o deseos localizables en el propio protagonista de la acción. Shütz (citado por Bertaux, 1997: 75) denomina al primer tipo de circunstancias causas porque (because), y al segundo tipo causas para qué (in order to).

La aproximación de Runyan (1984), ${ }^{3}$ con un fuerte acento individualista, descompone los hechos descritos en una narración en tres: a) procesos determinantes del comportamiento, b) procesos determinantes de la persona, y c) procesos determinantes de la situación. Los primeros hacen referencia a las situaciones en que las causas de los hechos narrados se pueden encontrar en la interacción de las personas con las circunstancias que las rodean, los segundos a las situaciones en que se ha producido un cambio en las características personales y los terceros a las situaciones en que las personas han influido en las circunstancias que las rodean. Runyan defiende que el análisis de estos tres tipos de procesos permite el examen de una deteminada secuencia biográfica o de la trayectoria biográfica completa. Debe destacarse que el elemento central del

\footnotetext{
3 Este autor apuesta por una combinación de métodos cuantitativos y cualitativos en el análisis biográfico. Por lo tanto, el análisis narrativo-biográfico constituye únicamente una parte de la aproximación metodológica de este autor.
} 
análisis lo constituye el primer tipo de procesos, puesto que según el autor son los que median (tanto en una dirección como en otra) entre los individuos y las situaciones por las que transcurre su biografía (1984: 85).

Wengraf (2000, 2001) comparte con Runyan la centralidad otorgada a la secuencialización de los datos biográficos como procedimiento para el análisis, aunque en otros aspectos difieren notablemente. El Método Biográfico Narrativo Interpretativo (BNIM) ${ }^{4}$ aplicado por el Centre for Biography and Social Policy y Wengraf (Chamberlayne y Spanò, 2000; Torrabadella, 2000; Wengraf, 2000; 2001) distingue en el análisis entre la "vida vivida" y la "vida narrada" aunque el procedimiento seguido otorga mayor centralidad a los datos atribuibles a la primera de estas dos instancias. El análisis de la vida vivida tiene un carácter fuertemente cronológico y se fundamenta en la secuencialización de los datos para encontrar la dinámica que ha caracterizado el caso analizado. Por su parte, la vida narrada se toma como elemento que permite detectar el sentido global dado a la narración y en su análisis se contemplan tanto aspectos de carácter sincrónico (ya apuntados) como diacrónico. El aspecto más interesante de esta propuesta de análisis es el detallado trabajo de identificación de todos los posibles desarrollos de la secuencia biográfica en un momento temporal dado. De este modo, el procedimiento permite identificar en cada punto biográfico concreto el campo de posibilidades abierto. En este análisis de posibles desarrollos, el/la analista intenta tomar la perspectiva del narrador, por lo tanto debe tener en cuenta tanto la información más estrictamente fáctica como la vinculada con la experiencia e identidad del individuo (Wengraf, 2001: 256-271).

\footnotetext{
${ }^{4}$ En algunas obras este método se denomina Método Biográfico Interpretativo (BIM).
} 
La tercera aproximación que situamos en este grupo de análisis preocupados fundamentalmente por la estructura diacrónica toma como elemento pivotal del análisis el concepto de acontecimiento. Aquí sitúo los trabajos de Legrand (1993) y de Leclerc-Olive (1997), autor y autora que pese a no haber colaborado en sus investigaciones tienen notables puntos de contacto. Tanto en uno como en otra, la aproximación al relato biográfico conserva muchos de los elementos de la investigación biográfica de la escuela de Chicago y de los trabajos desarrollados en Francia a partir de los años setenta, pero al mismo tiempo realizan un esfuerzo de distanciamiento del texto analizado por medio de la identificación de puntos o nodos biográficos de especial relevancia.

El trabajo de Leclerc-Olive (1997) se desarrolla fundamentalmente en torno a la idea de acontecimiento biográfico, entendiendo por ello aquellos puntos de la biografía en que la vida "da un cambio" (bascule) que marca todo el desarrollo posterior. Para la autora estos acontecimientos son centrales en la narración de cualquier biografía, es decir funcionan como balizamientos biográficos que marcan los puntos de paso obligados en cualquier relato biográfico. Según la autora estos acontecimientos tienen, además, un valor causal fundamental: una vez más la biografía no se entiende sin los vínculos causales entre los puntos de la biografía. ${ }^{5}$

Legrand (1993) parte también del concepto de acontecimiento, aunque su preocupación por las posibilidades de intervención biográfica (el autor es psicólogo) lo llevan a distanciarse de Leclerc-Olive en algunos puntos. El autor distinge entre acontecimientos activos y acontecimientos pasivos (destacando, de todos modos, que todo acontecimiento tiene a la vez aspectos activos y pasivos) y desarrolla categorías analíticas adicionales como las de coyuntura y catástrofe. El autor entiende por coyuntura un "tiempo intermedio de la biografía" (1993:163) que ejerce como elemento articulador entre las tendencias estructurales de larga duración (elementos de la biografía que escapan al control de las personas) y los acontecimientos puntuales que suceden en la biografía. Se trataría, por lo tanto, de situaciones de crisis o impasse en que se gesta el cambio en la trayectoria biográfica. El concepto de catástrofe hace referencia a los acontecimientos que irrumpen "accidentalmente" en la biografía y que producen una ruptura en la trayectoria biográfica. Por otro lado, en comparación con Leclerc-Olive, el análisis

\footnotetext{
${ }^{5}$ La autora afirma (1997: 91) haber elaborado para cada entrevista redes de conexión entre los diferentes acontecimientos narrados, aunque en la publicación consultada estas redes no se hacen públicas.
} 
de Legrand presta mayor atención a lo que denomina casi-acontecimientos (quasiévénements) entendiendo por ello aquellas acciones o situaciones biográficas (por ejemplo, una educación religiosa o una mala relación con los hermanos) que no tienen un carácter único y singular (característica de un acontecimiento en sentido estricto) pero que acaban influyendo en el rumbo biográfico y que, por lo tanto, retrospectivamente, son factores que explican una determinada trayectoria.

\section{Un modelo reticular para el análisis narrativo-biográfico}

Vistas en el epígrafe anterior las aproximaciones al análisis narrativo-biográfico que considero de mayor relevancia, en este epígrafe se apuntan brevemente los elementos principales del tipo de formalización reticular utilizada en Verd (2002). Esta aproximación, desarrollada en el marco del Análisis Reticular del Discurso, se distingue de otras versiones (véase por ejemplo Lozares en este mismo número o Martí, 2000) por el desarrollo de una estructura de caràcter tridimensional, que pretende abordar, simultáneamente, la estructura sincrónica y diacrónica presentes - como ya ha sido señalado- en cualquier texto narrativo.

\subsection{Una red de carácter tridimensional}

Como hemos comprobado en el epígrafe anterior, el número de aproximaciones cualitativas al análisis narrativo-biográfico en que se toma una actitud decidida de distanciamiento y objetivación del texto analizado son ya numerosas ( $y$ relativamente variadas). Ninguna de ellas, sin embargo, adopta un tipo de aproximación reticular (véanse, para una revisión en español, Lozares et al., 2003; Verd, 2005), pese a las analogías que permite esta aproximación tanto para el análisis de textos como para el análisis de acontecimientos biográficos - puesto que tanto unos como otros son fácilmente concebibles como nodos unidos por tipos específicos de relaciones. Ello se debe posiblemente al escaso conocimiento que se tiene aún hoy en día del análisis de redes textuales, término que traduce la expresión inglesa network text analysis (véanse, entre otros, Diesner y Carley, 2004; Carley, 1997; Popping, 2000; 2003). No obstante, debe señalarse que el análisis de redes textuales entronca claramente con las aproximaciones cognitivas al análisis de narraciones que se han mencionado en el punto 1, puesto que éstas tienen en esencia, una naturaleza reticular. 
Los detalles del procedimiento de construcción y análisis de las redes que están en la base de mi aproximación han sido ya tratados con anterioridad (Verd y Lozares, 2000; Verd, 2002; 2006) por lo que, por razones de espacio, no volveré a tratarlos aquí. Mencionar, no obstante, que el objetivo del procedimiento es el de conseguir traducir el texto de la narración biográfica a una red que se desarrolla en dos dimensiones diferentes: por un lado, mediante jerarquías de conceptos, muestra la dimensión cognitiva de los enunciados del narrador, y por otro lado, mediante relaciones de causalidad (no exclusivamente, pero sí principalmente) vincula en forma de secuencia los acontecimientos descritos en la narración. Ello implica recoger simultáneamente tanto la estructura sincrónica como la estructura diacrónica de la narración, lo cual se consigue mediante una doble codificación: cada segmento de texto es codificado a la vez situándolo en una estructura jerárquica y situándolo en relación con el conjunto de acontecimientos mencionados en la narración. Por lo tanto, una misma categoría (nodo) pertenece simultáneamente a dos redes diferentes, es por ello que se afirma que el resultado final del tratamiento de los datos es una red tridimensional.

El tipo de red que recoge la estructura sincrónica de la narración se inspira en los propios mapas semánticos (o redes semánticas) utilizados en las ciencias cognitivas. Concretamente, se utiliza una adaptación de las redes semánticas denominadas jerarquías de tipos, que buscan la representación de conceptos en forma de taxonomía jerárquica ordenada en función del nivel de generalidad (Sowa, 1991: 1). En este tipo de jerarquías se establece un sistema de pertenencias lógicas en que las categorías más concretas se asignan a las categorías más generales. Debe señalarse, además, que en este tipo de jerarquías las relaciones no tienen por qué tener un valor de verdad (truth value) y recogen únicamente las percepciones de los narradores (que, obviamente, cambian de una persona a otra). Las gráficas 1 y 2 presentan las redes semánticas para una misma categoría (primer empleo) en el caso de dos personas entrevistadas diferentes (Cesca y Miguel). Puede comprobarse que esta categoría pertenece a una categoría más abstracta denominada trayectoria laboral, la cual, en los dos casos, se subdivide en el nivel inmediatamente inferior en las categorías primer empleo y segundo empleo (la jerarquía correspondiente a segundo empleo no se ha desarrollado en ninguno de los dos ejemplos). 
El tipo de red que recoge la estructura diacrónica de la narración ${ }^{6}$ es la que más propiamente se inspira en el análisis de redes textuales. En esta red las categorías (fruto de la codificación de los acontecimientos narrados en el relato biográfico) se relacionan mediante relaciones de causalidad, de identidad y de oposición. Las gráficas 3 y 4 recogen las redes biográficas de este tipo para los mismos casos correspondientes a las gráficas 1 y 2 . En ellas puede observarse que las relaciones de causalidad se han representado mediante el símbolo "=>", las relaciones de identidad mediante el símbolo "==" y las relaciones de oposición mediante el símbolo "==--". Como se comprueba, la mayoría de relaciones (recordemos que son las establecidas por los narradores en sus relatos) son de causalidad, algo que, como se ha señalado más arriba, es característico de todo tipo de narraciones.

Este segundo tipo de red es la que más propiamente supone una aplicación del modelo metodológico de redes sociales, y como tal, la que permite calcular e identificar todo un conjunto de indicadores de carácter reticular que en el caso de las redes que tratamos tiene un sentido biográfico. El repaso a estos indicadores se realiza en la siguiente sección. La práctica totalidad de los indicadores que se propondrán podrían ser calculados mediante la conversión de los grafos en matrices y con posterioridad ser analizados mediante un programa estadístico de tratamiento de redes, sin embargo, este conversión no se ha realizado, puesto que el tamaño relativamente pequeño de las redes biográficas no lo hace necesario; además, el trabajo con matrices ${ }^{7}$ únicamente añadiría un estadio más al proceso de tratamiento de los datos, sin producir ningún beneficio adicional.

\footnotetext{
${ }^{6}$ Estas redes se han elaborado utilizando el programa ATLAS.ti.

${ }^{7}$ Este trabajo exigiría, además, la construcción de una red diferente para cada uno de los tres tipos de relaciones representadas. Ello no se ha llevada a cabo puesto que esta disgregación volvería a segmentar el discurso de los/as entrevistados/as, que es lo contrario de lo que pretende la representación gráfica. Los indicadores utilizados se han calculado en base a esta representación heterodoxa, por lo tanto tampoco hacen la distinción entre los diferentes tipos de relación.
} 


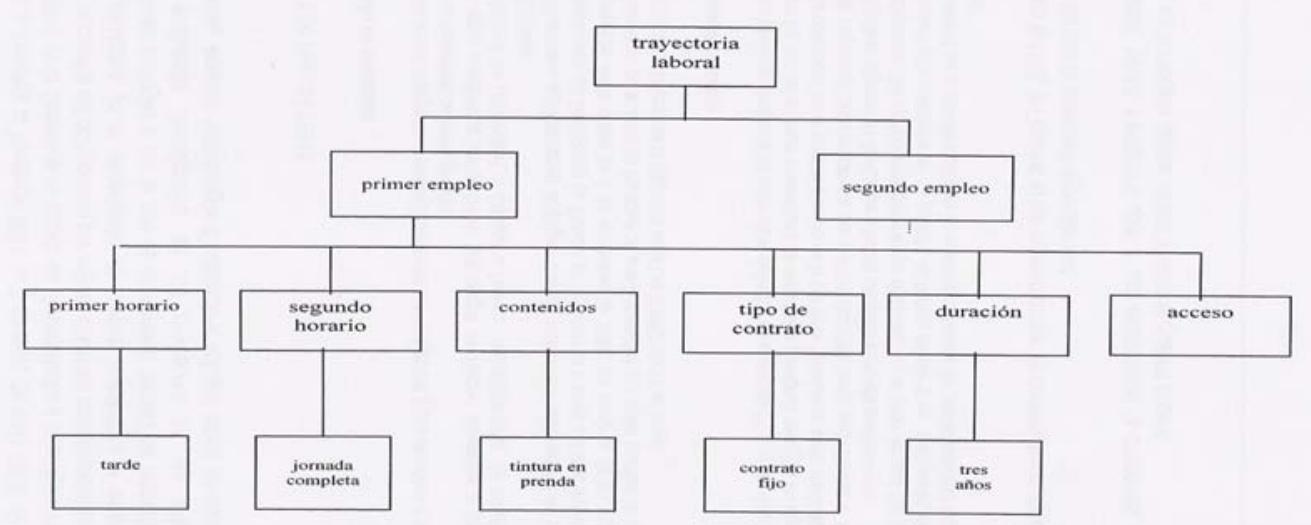

I lustración 1. Red semántica de la categoría primer empleo en la narración de Cesca. Fuente: elaboración propia a partir de Verd, 2002: 288-289

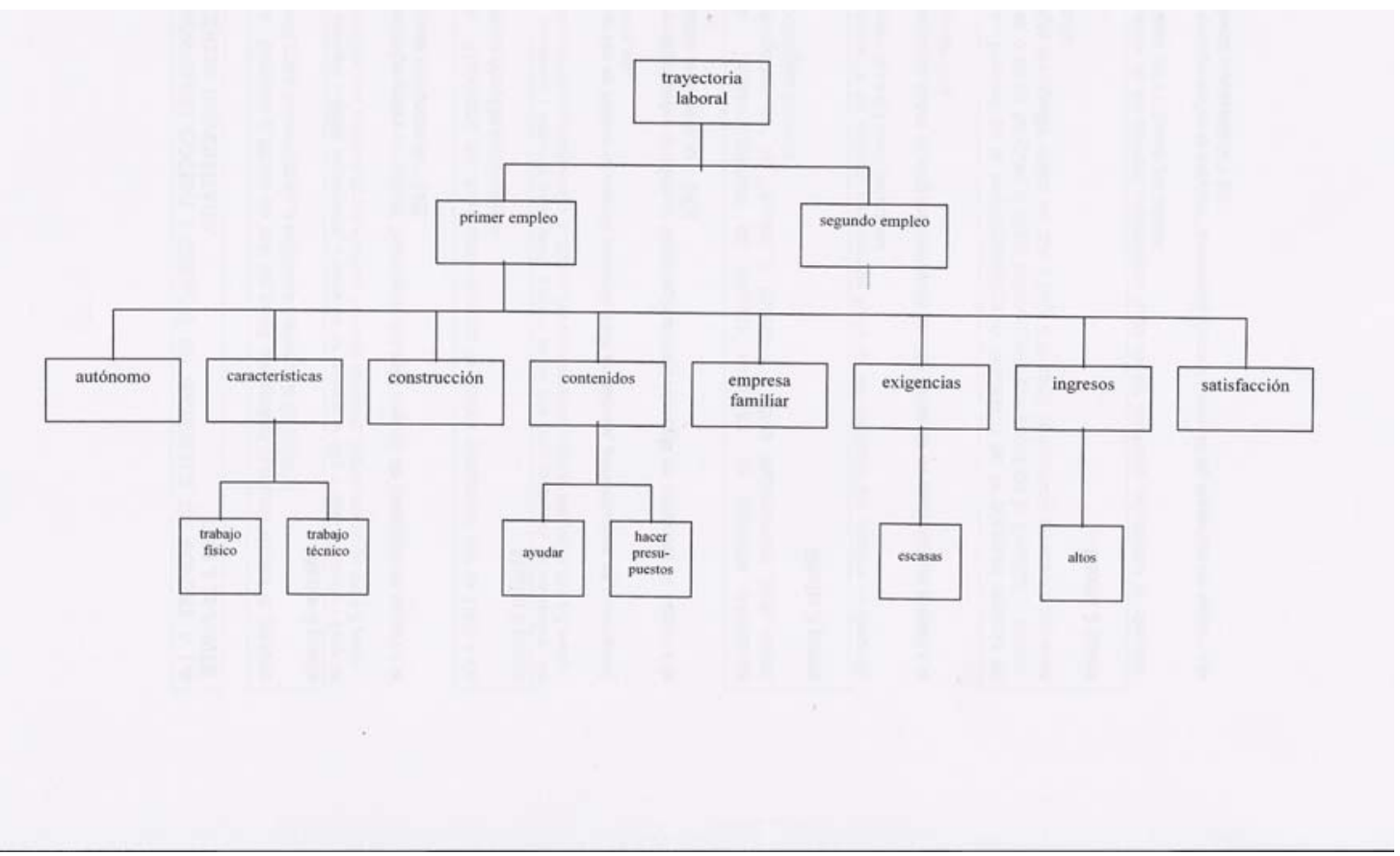

I lustración 2. Red semántica de la categoría primer empleo en la narración de Miguel. Fuente: elaboración propia a partir de Verd, 2002: 304-305 


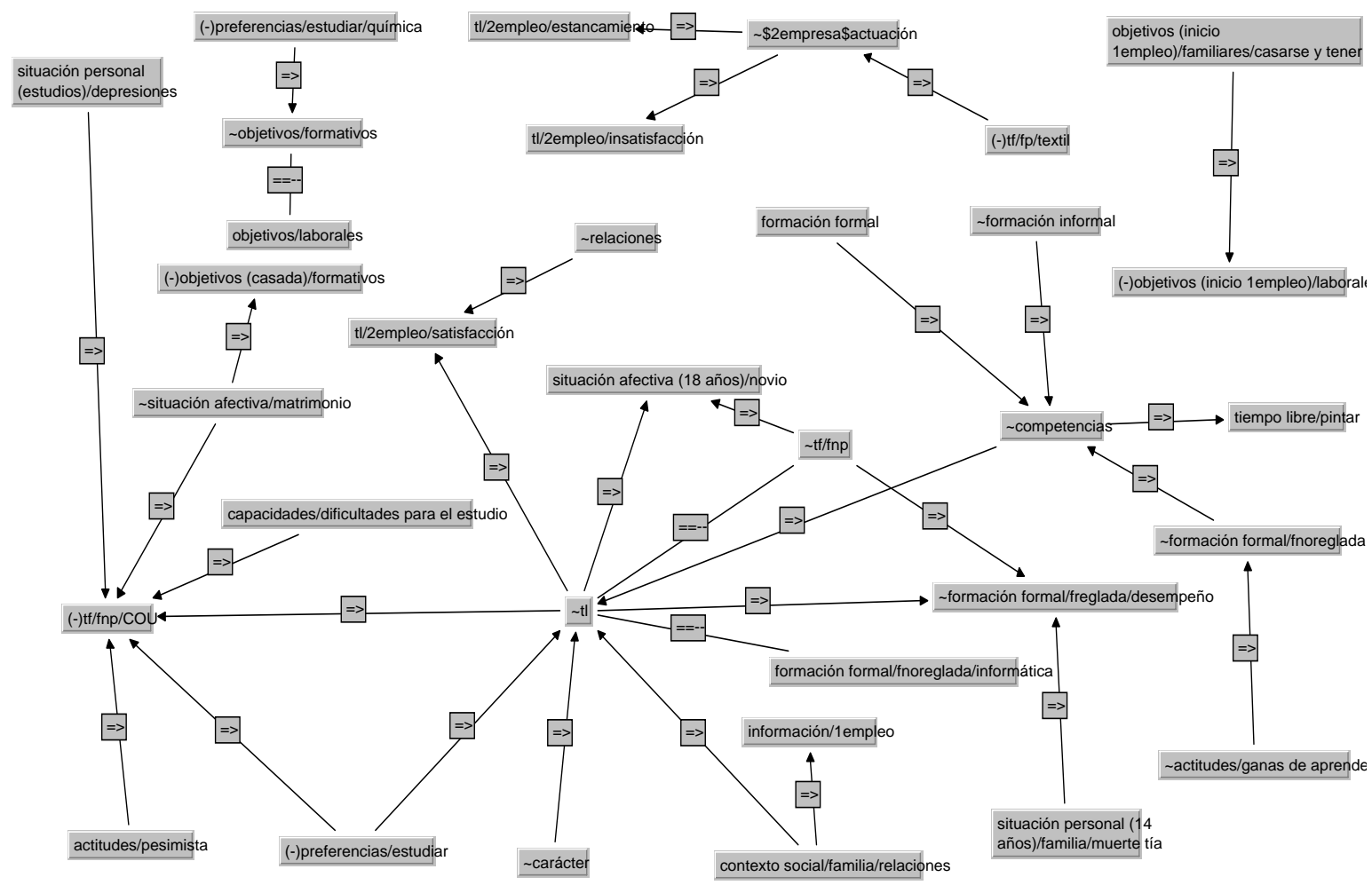

I lustración 3. Red biográfica de Cesca. Estructura diacrónica. Fuente: Gráfica IX.1 (Verd, 2002:

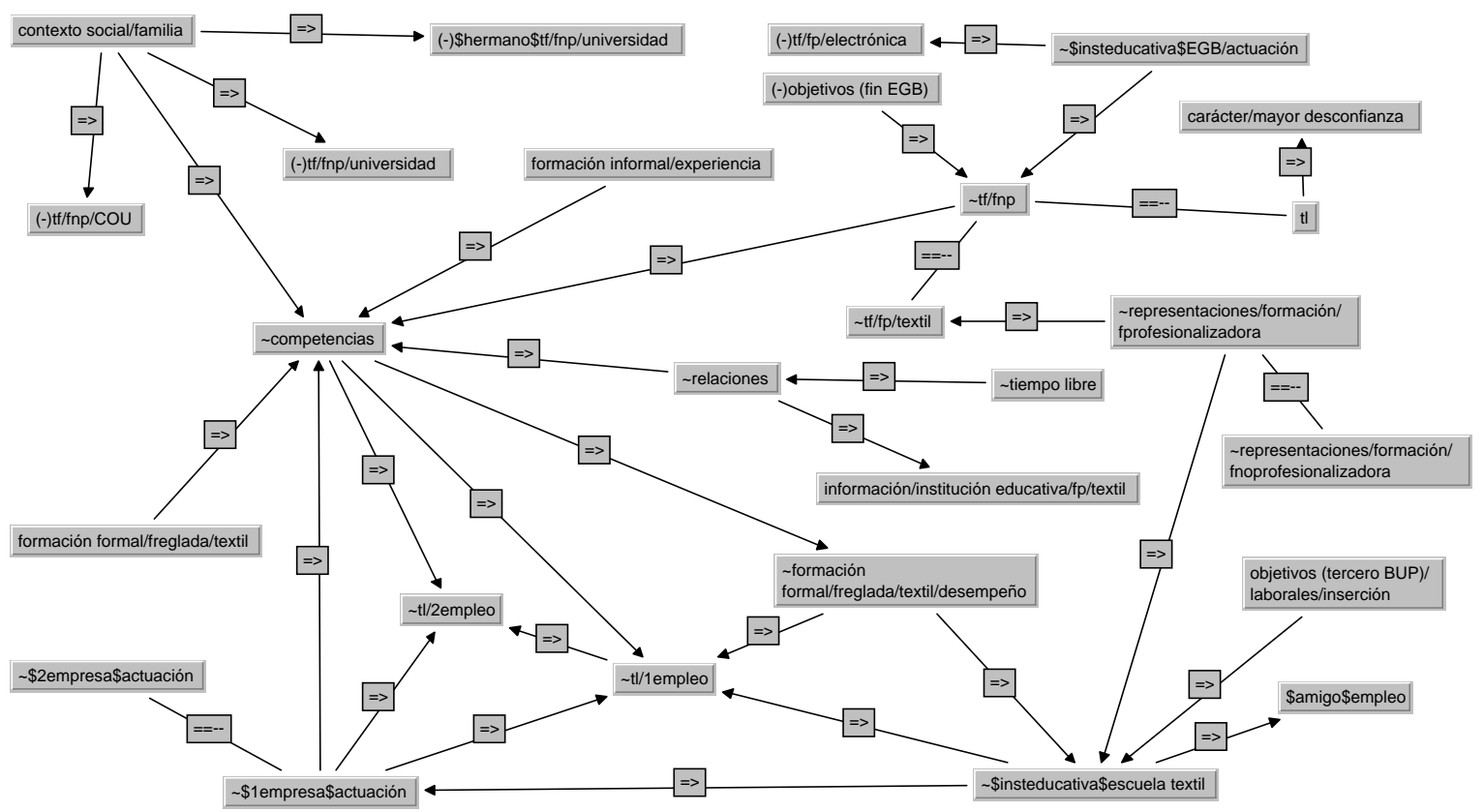

I lustración 4. Red biográfica de Miguel. Estructura diacrónica. Fuente: Gráfica IX.5 (Verd, 2002: 


\subsection{Indicadores biográficos a que da lugar el procedimiento}

Como es sabido, buena parte de los indicadores de redes sociales provienen de la teoría de grafos, que no sólo ha suministrado una forma de visualización de las redes, sino que tomada como teoría matemática ha proporcionado también conceptos y teoremas para muchos de los análisis de redes sociales. En la propuesta de análisis que aquí se presenta este cálculo de indicadores supone el último estadio de tratamiento de los datos, de modo que una vez construidas las redes biográficas se ha procedido a calcular e identificar un conjunto de indicadores que sintetizan y permiten objetivar la información representada gráficamente. Además, estos indicadores ejercen de vínculo entre los elementos biográficos del texto, o empiria, y los diferentes niveles de abstración con que se trabaje en los conceptos o teorías. El tipo de indicadores que se calculan son de carácter relacional -tomando la distinción que hace Burt (1980) entre enfoques relacionales y posicionales- y se han designado con la terminología tradicional en redes sociales (Iacobucci, 1994). El pequeño fragmento de red biográfica representado en la gráfica 5 servirá para ejemplificar los diferentes cálculos. La aplicación práctica de estos indicadores y los resultados a que da lugar su uso se pueden consultar en Verd (2002), aquí únicamente se explicará su sentido más general.

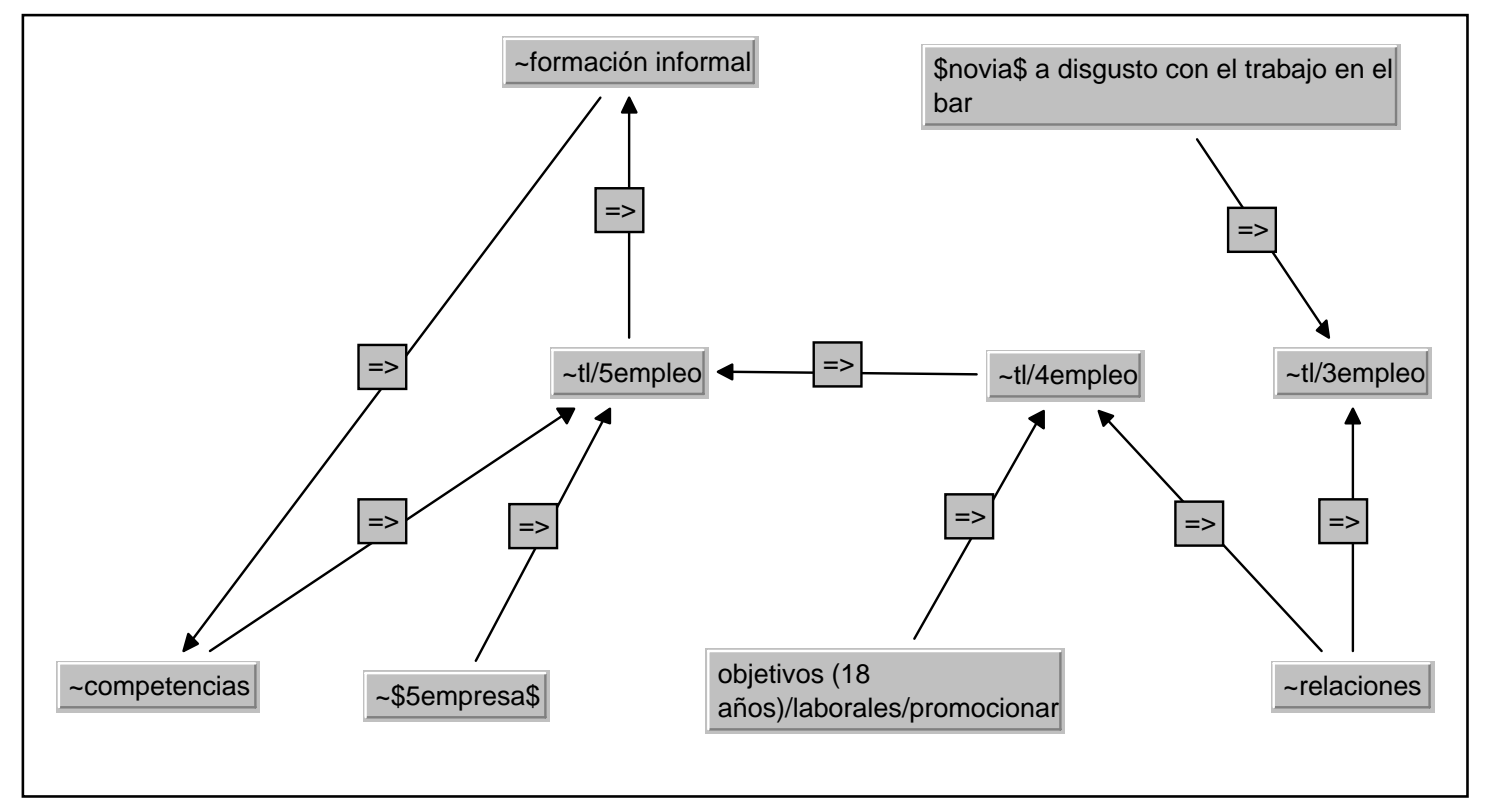

I lustración 5. Fragmento de red biográfica (J ulio). Fuente: Gráfica VII.5 (Verd, 2002: 248) 
El primero de los indicadores que tiene sentido en el análisis de una red biográfica es el grado nodal. Se entiende como tal el número de relaciones que cada nodo recibe o transmite de/hacia el resto de nodos de la red. Suele representarse mediante el símbolo $d$ (del término inglés degree) y en las redes que se han analizado equivale a la suma de todos los tipos de relación que establece el nodo considerado. De este modo, en la gráfica 5 el nodo tl/5empleo (quinto empleo en la trayectoria laboral) tiene un $d=4$ y el nodo tl/3empleo (tercer empleo en la trayectoria laboral) un $d=2$.

En las redes dirigidas es posible calcular el grado nodal de entrada y grado nodal de salida (en inglés indegree y outdegree, respectivamente), representados por los símbolos $d_{1}$ y $d_{0}$. El grado nodal de entrada se calcula sumando el número de relaciones en las cuales el nodo considerado es el receptor; y el grado nodal de salida sumando el de las relaciones en que el grado considerado es el emisor. A partir del grado nodal de entrada y de salida es posible identificar en una red biográfica los transmisores puros y los receptores puros. Los primeros son aquellos nodos en que $d_{1}=0$ y $d_{0}>0$ y los segundos aquellos en que $d_{1}>0$ y $d_{0}=0$. En la gráfica 5 el nodo tl/3empleo es un receptor puro y el nodo competencias un transmisor puro.

El cálculo del grado nodal absoluto y del de entrada y de salida permite obtener una primera base para establecer la importancia de cada uno de los acontecimientos biográficos representados por los nodos. El análisis en Verd (2002) se ha dirigido concretamente hacia la explicación del papel jugado por los nodos de mayor grado nodal en cada red, así como por el de los trasmisores puros y los receptores puros. Un elevado grado nodal implica un papel preponderante en la trayectoria biográfica por parte del acontecimiento representado: ya sea porque se trate de un suceso que ha marcado el desarrollo ulterior de los hechos, ya sea porque es el fruto de un cúmulo de circunstancias, el acontecimiento con un elevado grado nodal tiene en la concepción del narrador una importancia destacada. ${ }^{8}$ Un nodo que es transmisor puro (de un número mínimo de relaciones) tiene en la narración el papel de principio generador, de causa última que explica el desarrollo de los

\footnotetext{
${ }^{8}$ Como ya habrá advertido el lector en la explicación de todo el procedimiento, el número de veces que un acontecimiento aparece citado -y por lo tanto codificado- a lo largo de la narración no tiene ninguna relevancia en el análisis practicado. Tiene muy poco sentido considerar esta vertiente "cuantitativa" en textos que son fruto de una entrevista, puesto que las reiteraciones o repeticiones de acontecimientos obedecen mucho más al estilo de narración y al desarrollo de la entrevista que a una supuesta importancia otorgada por el narrador. La consideración de las relaciones elimina este "efecto de repetición" midiéndose la importancia de un acontecimiento por la posición como situación causante y causada en el conjunto de la trayectoria (biográfica) descrita.
} 
acontecimientos posteriores. Por otro lado, un nodo que es receptor puro (de un número mínimo de relaciones) tiene el sentido de meta o finalidad conseguida, aunque a veces también de "callejón sin salida" o de "posibilidad no practicada".

Otro tipo de nodos con unas características especiales y que es preciso considerar en el análisis son los denominados puntos de corte. Un punto de corte es un nodo que si se elimina de la representación deja desconectados a un conjunto de nodos que anteriormente formaban parte del mismo grafo. No existen medidas estándar para calibrar la importancia de un punto de corte. En la aproximación que presento he calculado esta importancia multiplicando el número de nodos que contiene cada uno de los subgrafos producidos. Es decir cuanto mayor sea el tamaño de los subgrafos producidos y menor sea la diferencia existente entre ellos, mayor será la importancia del punto de corte. ${ }^{9}$ Así en la gráfica 5 el punto de corte de mayor importancia es $\mathrm{tl} / 4 \mathrm{empleo}$ (cuarto empleo en la trayectoria laboral), puesto que el producto del tamaño de los subgrafos generados $(3 \times 1 \times 4=12)$ es mayor que el producto del tamaño de los subgrafos generados al eliminar tl/3empleo (el resultado es $7 \times 1=7$ ) o tl/5empleo (el resultado es $2 \times 1 \times 5=10$ ). El sentido de estos puntos de corte en la narración suele ser el de intermediación o delimitación de diferentes "mundos" del actor, de diferentes áreas en la vida del/de la entrevistado/a.

Finalmente, se ha utilizado en el análisis un indicador que no hace referencia a los nodos, sino a los arcos (lazos que unen los nodos) y que puede considerarse el análogo al punto de corte. Se trata del concepto de puente. Un arco se considera un puente si su eliminación de la representación deja desconectados a un conjunto de nodos que anteriormente estaban conectados. La importancia de un puente se ha medido del mismo modo que se ha hecho en el caso de los puntos de corte, es decir multiplicando el tamaño de los subgrafos generados con su eliminación. En el caso de la gráfica 5 el puente de mayor importancia es el que une los nodos tl/4empleo y tl/5empleo, puesto que el producto del tamaño de los dos subgrafos generados $(5 \times 4=20$ ) es el mayor que puede obtenerse de realizar la multiplicación indicada entre subgrafos situados a lado y lado de los puentes existentes.

9 Esta medida se aproxima al cálculo de la centralidad de intermediación (betweemess centrality) que puede definirse intuitivamente como la capacidad que tiene un actor (el nodo que lo representa) de poner en contacto el mayor número de subgrafos que de otro modo no tendrían contacto directo (véase Wasserman y Faust, 1994: 189-191). 
Evidentemente, al lado del cálculo de estos indicadores y su interpretación, el análisis de las narraciones biográficas exige la consideración de todo el conjunto de nodos y relaciones representadas en la red. Tanto el tamaño de los grafos y subgrafos (es decir el número de nodos que constituyen bloques biográficos completos) como el contenido concreto de las situaciones o acontecimientos representados en los nodos son informaciones fundamentales en la interpretación de las narraciones biográficas. Por lo tanto, el análisis biográfico que se realiza es el resultado, en primer lugar, de la traducción de la narración a una red biográfica tridimensional (en que se deben interpretar tanto las estructuras sincrónicas como diacrónicas), en segundo lugar, del contenido y sentido biográfico de todos los acontecimientos identificados como relevantes por parte del/de la narrador/a, y, en tercer lugar, de la identificación de los puntos clave (nodos con mayor relevancia) en la trayectoria descrita. El objetivo de todo ello es mejorar (respecto a los análisis puramente temáticos o descriptivos) el tipo de análisis realizado.

\section{Aportaciones de la aproximación al análisis narrativo- biográfico}

La aproximación al análisis de narraciones biográficas que se ha presentado en las páginas precedentes pretende ser una contribución al análisis narrativo-biográfico en que el tipo de formalización llevada a cabo contribuya al rigor y la sistematización de la ulterior reflexión teórica. Se trata de un procedimiento que es explícto y transparente, y que permite también la reproducción y la contrastación del proceso de análisis, de modo que es posible evaluar la corrección no sólo de las diferentes etapas, sino también de las propias inferencias de carácter teórico extraídas. Las redes, con su doble estructura sincrónica y diacrónica, ponen de manifiesto cómo han sido interpretados (y simplificados) los textos. La formalización reticular permite, además, una superación de los análisis exclusivamente temáticos o tipológicos, preponderantes aún hoy en día en los análisis cualitativos de textos.

Por otro lado, la traducción del relato biográfico a una red de acontecimientos (representados por los nodos) y relaciones entre ellos supone la posibilidad de identificar empíricamente los principales hechos ocurridos a lo largo de la trayectoria biográfica, sin necesidad de tener que acudir a la "opinión" retrospectiva de la persona entrevistada. Esta afirmación se entenderá mejor con un ejemplo. El fragmento de entrevista que figura a continuación se ha extraido de la entrevista a Cesca - cuya red biográfica se ha presentado en la gráfica 1 . Se sitúa en el 
contexto de un conjunto de preguntas sobre la interrupción de sus estudios en COU y su repercusión posterior en su trayectoria laboral:

Pregunta: ¿La necesidad de estudiar es una preocupación que te persigue o... o no?

Respuesta: No, no, no. No me preocupa, o sea yo ahora mismo, yo me propuse llegar hasta tercero de BUP, ¿no? O sea, me preocupa y no me preocupa, porque ahora, ahora sí que me preocupa porque pienso ves, si yo tuviera un estudio yo podría decir, pues mira, aquí os quedáis que me busco otro, otro trabajo, ¿no?

Se trata del fragmento de toda la entrevista en que más explícitamente se realiza una reflexión sobre las consecuencias de haber dejado los estudios. Como se comprueba, la entrevistada muestra una actitud de duda que permite sospechar que sí considera que su interrupción ha tenido efectos negativos en su trayectoria laboral, aunque no se trata de una afirmación categórica. Sin embargo el análisis de la red biográfica (y por lo tanto del conjunto de acontecimientos más destacados en su trayectoria biográfica) permite comprobar la enorme importancia que en su narración tiene la interrupción de los estudios. Esta importancia la muestra el hecho de que el nodo que representa esta interrupción ((-)tf/fnp/COU), además de tener un elevado grado nodal, es el receptor puro más importante de la red ${ }^{10}$-en realidad el que tiene mayor grado nodal de entrada de todas las redes biográficas analizadas en Verd (2002)- lo cual pone de manifiesto la importancia estructural del acontecimiento. Se trata en realidad de "algo que no pasó" que marca un punto de bifurcación en la vida de la entrevistada; marca un camino que no siguió pero que visto desde el presente tenía una importancia notable.

El ejemplo precedente muestra en qué medida los indicadores estructurales obtenidos son útiles para identificar los jalones biográficos más representativos sin necesidad de "hacer escoger" a las personas entrevistadas, lo cual podría implicar un sesgo racionalizador o naturalizador por parte del narrador. La aproximación que se ha presentado no sólo permite reconstruir las acciones pasadas, sino también detectar a partir de su posición estructural en el relato cuáles de ellas son más relevantes. Utilizando este método la importancia de un acontecimiento viene indicada por el número de relaciones que tiene el nodo que lo representa, es decir

\footnotetext{
${ }^{10}$ Estas afirmaciones pueden comprobarse observando la llustración 1.
} 
por el número de implicaciones/influencias que ha tenido ese suceso en el resto de hechos narrados.

Esta centralidad de los nodos viene propiciada por la necesidad cognitiva que tienen los narradores de aportar una explicación a los cambios o giros biográficos o de explicar las consecuencias de un accidente biográfico: cuanto mayor es el número de razones esgrimidas como causa del giro o mayores las consecuencias inesperadas causadas, mayor es el grado nodal del acontecimiento descrito (en el primer caso se trata de un elevado grado nodal de entrada y en el segundo caso de un elevado grado nodal de salida).

Una de las consecuencias de este balizamiento de la biografía mediante "puntos clave" es la posibilidad de describir la trayectoria biográfica como un conjunto de acontecimientos biográficos encadenados (en el sentido en que entienden este término Leclerc-Olive o Legrand). Ello permite representar gráficamente la "espina dorsal" de la narración, que en términos formales está constituida por los nodos con mayor grado nodal y los lazos existentes entre ellos.

En realidad, el tipo de análisis seguido no hace más que dotar de contenido empírico (y de un procedimiento formal y explícito para identificarlos) a un conjunto de conceptos que tienen ya larga tradición en los análisis narrativo-biográficos. Las ideas de encrucijada, de bifurcación o de "punto de no retorno" son constantes en los análisis biográficos. Como nos recuerda Leclerc-Olive, "en los relatos biográficos los acontecimientos son muy a menudo momentos de intermediación, momentos de 'bifurcación'" (1997: 31); por su parte, con un perspectiva teórica algo diferente, Lahire señala la importancia de "hacer hablar a los momentos de "ruptura biográfica", de cambios o de modificaciones, incluso ligeros, en las trayectorias o las carreras [...] puesto que son los momentos en que las disposiciones pueden ser puestas en cuestión o pueden ser repentinamente reactivadas cuando hasta entonces habían estado en estado de vigilia" (2002: 3031, con cursivas en el original). Estos puntos de ruptura o de bifurcación coinciden en las redes, en buena medida, con los nodos de mayor grado nodal. Además, como ha sido señalado, si estos nodos son transmisores puros o receptores puros, su sentido es, respectivamente, de inicio o final de una etapa -que no tiene solución de continuidad con los hechos anteriores o posteriores. 
La representación en forma de red de los acontecimientos vividos permite, por lo tanto, una identificación empírica de los puntos biográficos en que efectivamente se producen dichas situaciones de intermediación o bifurcación. En algunos casos se trata de la piedra angular o clave de arco de la narración analizada, en otros casos se trata de un problema, de una dificultad que marca los acontecimientos posteriores. En general, pues, estos puntos corresponden a situaciones que han condicionado el curso de las acciones y acontecimientos posteriores. De todos modos, algunos de estos nodos centrales en la narración se corresponden más con el término casi-acontecimiento de Legrand (1993). En algunas trayectorias biográficas los nodos más centrales no siempre son puntos de bifurcación, a veces son puntos de movilización o adquisición de recursos. En estos casos definen los acontecimientos posteriores no por ruptura o accidente sino por saturación, son puntos de continuidad que marcan únicamente un pico dentro de una serie de acontecimientos que siguen una misma lógica, no se trata pues, de sucesos irrepetibles y fuertemente localizados en el tiempo que hayan cambiando el rumbo biográfico; sí son, no obstante, elementos clave de la biografía que explican la trayectoria seguida.

Resulta más difícil encontrar en la literatura una correspondencia teórica a las situaciones identificadas mediante los puntos de corte y los puentes. Se trata en general de situaciones cuya posición en la narración tiene más que ver con un tipo de acontecimiento que une y da coherencia a todo el relato, por lo tanto constituyen también, en cierto modo, la espina dorsal del relato, aunque en este caso sin el valor de "pico biográfico". En estos nodos (o lazos) aquello que resulta más relevante son las diferentes "parcelas" o "fases" biográficas que quedan desconectadas (subgrafos) si el punto de corte o el puente es eliminado.

Para terminar, es interesante señalar que a mayor grado de "narratividad" en el texto analizado mayor ha sido la densidad y conectividad de los grafos. Ello demuestra el grado de adecuación de la formalización reticular utilizada a los contenidos de entrevistas narrativas (no necesariamente biográficas), pero también las dificultades de aplicación del mismo tipo de formalización a entrevistas que no tengan ese componente narrativo. Ello se explica precisamente porque la forma narrativa permite dar coherencia y una línea explicativa a todo un conjunto de hechos; en la medida en que estos mismos hechos se presenten utilizando otra forma lingüística menores son las conexiones verbalizadas. En términos de redes ello supone que cuanto menos se cumplen las características que lingüísticamente 
permiten identificar a un texto como narrativo, mayor es el número de subgrafos de la red (menor conexión existe entre diferentes hechos) y menor es su tamaño.

\section{Conclusiones}

La aproximación al análisis de narraciones sociobiográficas que se ha presentado en las páginas precedentes pretende conjugar el examen tanto de la estructura sincrónica como de la estructura diacrónica que está presente en toda narración. No obstante, en el presente artículo se ha hecho incidencia fundamentalmente en el segundo tipo de estructura, la que recoge el conocimiento episódico de las personas. Siguiendo un procedimiento inspirado en el análisis reticular del discurso se ha propuesto el uso de todo un conjunto de medidas que permiten identificar los puntos clave en las trayectorias biográficas descritas.

La narración biográfica es al mismo tiempo una descripción de acontecimientos vividos y una "explicación" de la situación biográfica presente (asociada fuertemente a la propia identidad). Es una explicación desde la subjetividad, y en ese sentido fruto claramente de la apropiación cognitiva que el narrador ha hecho de los acontecimientos narrados: "Una narración es el proceso cognitivo que otorga significado a acontecimientos de carácter temporal mediante su identificación como partes de una misma trama [plot]" (Polkinghorne, citado por Neisser y Libby 2000: 318). Esta "coherencia narrativa" de los hechos es la que permite representar en una única estructura reticular todo el conjunto de hechos narrados; de todos modos el sentido dado por el sujeto a su narración no tiene por qué ser necesariamente el mismo que se extrae del análisis de los hechos narrados, frecuentemente la realidad es suficientemente tozuda como para contradecir o poner en duda el sentido dado por el narrador a esos hechos.

Precisamente una aproximación formalizada que objetive y permita tomar distancia respecto al discurso identitario (lo cual no implica que no deba tenerse en cuenta para la interpretación de la narración, de igual modo que deben considerarse todo el conjunto de contextos que intervienen en la elaboración de un discurso) permite un trabajo sistemático de cuestionamiento y análisis de las conexiones entre hechos expuestas en la narración biográfica. La aproximación reticular al análisis de narraciones que se ha presentado, fundamentada principalmente en "relaciones de causalidad", ofrece esta posibilidad de identificación empírica de los acontecimientos principales en una trayectoria biográfica dada. 
Este tratamiento reticular permite una reducción teóricamente guiada de la información analizada y la presentación gráfica de los datos (es decir, de la información finalmente retenida para cada una de las narraciones) de un modo fácilmente comprensible. Los indicadores obtenidos con esta aproximación reticular se corresponden, además, con ciertos conceptos teóricos utilizados en otras aproximaciones metodológicas al análisis narrativo-biográfico.

Para finalizar, mencionar también las potencialidades que ofrece el método seguido en los casos en que se desea realizar un análisis comparativo entre diferentes narraciones. Las posibilidades de simplificación de las trayectorias biográficas y la facilidad de focalizar el análisis en unos acontecimientos o periodos biográficos concretos permiten establecer diferentes niveles de comparación, desde los más globales a los más concretos, gracias a los diferentes niveles de abstracción a los que puede establecerse el análisis. Se trata, en definitiva, de aprovechar la multidimensionalidad de los datos narrativo-biográficos para acometer el estudio de las biografías en toda su complejidad.

\section{Bibliografía}

Abell, P. (1987). The Syntax of Social Life. Oxford: Clarendon Press.

- (1988). The 'structuration' of action. Inference and comparative narratives, en Nigel G. Fielding (ed.): Actions and Structure. Research Methods and Social Theory. Londres: Sage.

Adam, J.-M. (1992). Les textes: types et prototypes. París: Nathan.

Bell, J. (2002/1999). Cómo hacer tu primer trabajo de investigación. Guía para investigadores en educación y ciencias sociales. Barcelona: Gedisa.

Bertaux, D. (1997). Les récits de vie. París: Nathan.

Bruner, J. (1987). "Life as Narrative”, Social Research, 54 (1): 11-32.

- (1991). "The Narrative Construction of Reality", Critical Inquiry, 18: 1-21.

Burt, R.S. (1980). "Models of network structure", Annual Review of Sociology, 6: 79-141.

Carley, K. (1997). "Network text analysis: The network position of concepts", en Carl W. Roberts (ed.): Text Analysis for the Social Sciences: Methods for Drawing Statistical Inferences from Texts and Transcripts. Mahwah, New Jersey: Lawrence Erlbaum.

Calsamiglia, H. y Tusón, A. (1999). Las cosas del decir. Manual de análisis del discurso. Barcelona: Ariel. 
Chamberlayne, P. y Spanò, A. (2000). “Modernisation as lived experience: contrasting case studies from the SOSTRIS project". En Prue Chamberlayne, Joanna Bornat y Tom Wengraf (ed.): The Turn to Biographical Methods in Social Science. Comparative Issues and Examples. Londres: Routledge, pp. 321-336.

Demazière, D. y Dubar, C. (1997). Analyser les entretiens biographiques. L'exemple de récits d'insertion. París: Nathan.

Diesner, J. y Carley, K. M. (2004). “Using Network Text Analysis to Detect the Organizational Structure of Covert Networks". En Proceedings of the North American Association for Computational Social and Organizational Science (NAACSOS) 2004 Conference, Pittsburgh.

Dijk, T. A. van (1999/1998). Ideología. Una aproximación multidisciplinar. Barcelona: Gedisa.

Dyer, M. G. (1983). "The Role of Affect in Narratives", Cognitive Science 7: 211242.

Fielding, N. G. y Lee, R. M. (1998). Computer Analysis and Qualitative Research. Londres: Sage.

Gergen, K. J. (1996/1994). Realidades y relaciones. Aproximaciones a la construcción social. Barcelona: Paidós.

Greimas, A. J. (1971/1966). Semántica estructural. Madrid: Gredos.

Heise, D. R. (1991). “Event Structure Analysis: A Qualitative Model of Quantitative Research". En Nigel G. Fielding y Raymond M. Lee (eds.): Using Computers in Qualitative Research. Londres: Sage.

Iacobucci, D. (1994). "Graphs and Matrices". En S. Wasserman y K. Faust: Social Network Analysis. Cambridge: Cambridge University Press.

Labov, W. S. (1997). "Some Further Steps in Narrative Analysis", Journal of Narrative and Life History, 7: 395-410.

- (2000/1972). The transformation of experience in narrative. En Adam Jaworski y Nikolas Coupland (comp.): The Discourse Reader. Londres/Nueva York: Routledge.

Labov, W.S. y Waletsky, J. (1967). "Narrative Analysis: Oral Versions of Personal Experience". En J. Helm (comp.): Essays on the verbal and visual arts. Seattle: University of Washington Press, pp. 12-44.

Lahire, B. (2002). Portraits sociologiques. Dispositions et variations individuelles. París: Nathan.

Larsen, P. (1993). “Análisis textual del contenido de ficción de los medios de comunicación". En K. B. Jensen y N. W. Jankowski (eds.): Metodologías cualitativas de investigación en comunicación de masas. Barcelona: Bosch.

Leclerc-Olive, M. (1997). Le dire de l'événement (biographique). Lille: Presses Universtaires du Septentrion.

Legrand, M. (1993). L'approche biografique. París: Desclée de Brouwer. 
Lehnert, W. G. (1981). "Plot Units and Narrative Summarization", Cognitive Science 4: 293-331.

Lozares, C.; Verd, J.M.; Martí, J y López, P. (2003). "Relaciones, redes y discurso: revisión y propuestas en torno al análisis reticular de datos textuales", Revista Española de Investigaciones Sociológicas, 101: 175-200.

Manning, P. K. y Cullum-Swan, B. (1994). "Narrative, Content and Semiotic Analysis". En Norman K. Denzin e Yvonna S. Lincoln (eds.): Handbook of Qualitative Research. Thousand Oaks, California: Sage.

Martí, J. (2000). Formació i ocupació en el discurs dels treballadors. Una proposta metodològica. Universitat Autònoma de Barcelona, Departamento de Sociología. Tesis Doctoral.

Miles, M. B. y Huberman, A. M. (1994). Qualitative Data Analysis. An Expanded Sourcebook. Thousand Oaks, California: Sage.

Neisser, U. y Libby, L. K. (2000). "Remembering Life Experiences". En Endel Tulving y Fergus I. M. Craik (ed.): The Oxford Handbook of Memory. Oxford/New York: Oxford University Press.

Popping, R. (2000). Computer-assisted Text Analysis. London: Sage.

- (2003). "Knowledge graphs and network text analysis", Social Science Information, 42: 91-106.

Propp, V. (1977/1968). Morfología del cuento. Madrid: Fundamentos.

Ramos, R. (2001). Narrativas contadas, narraciones vividas. Un enfoque sistémicode la terapia narrativa. Barcelona: Paidós.

Renkema, J. (1999/1993). Introducción a los estudios sobre el discurso. Barcelona: Gedisa.

Riessman, C. K. (1993). Narrative Analysis. Newbury Park, California: Sage.

Runyan, W. M. (1984). Life Histories and Psychobiography. Explorations in Theory and Method. New York/Oxford: Oxford University Press.

Schegloff, E. A. (1997). “'Narrative Analysis' Thirty Years Later", Journal of Narrative and Life History, 7: 97-106.

Sowa, J. F. (1991). "Issues in knowledge representation", en John F. Sowa (ed.): Principles of Semantic Networks. Explorations in the Representation of Knowledge. San Mateo, California: Morgan Kaufmann.

Torrabadella, L (2000). “El «mètode biogràfic interpretatiu». Una eina per a la imaginació sociològica", Revista Catalana de Sociologia, 11: 133-152.

Verd, J, M. (2002). Itinerario biográfico, recursos formativos y empleo. Una aproximación integrada de carácter teórico y metodológico. Universitat Autònoma de Barcelona, Departament de Sociologia. Tesis Doctoral. (Puede consultarse el texto en http://www.tdr.cesca.es/index_tdx_cs.html) 
- (2005). "El uso de la teoría de redes sociales en la representación y análisis de textos. De las redes semánticas al análisis de redes textuales", Empiria. Revista de Metodología de Ciencias Sociales, 10: 129-150.

- (2006 [en prensa]). “Análisis de narraciones sociobiográficas: una visión reticular, cognitiva y social", en Carlos Lozares (ed.): Redes sociales y cognición. Granada: Comares.

Verd, J. M. y Lozares, C. (2000). "La teoria de xarxes socials aplicada a la interpretació d'entrevistes narratives. Una proposta", Revista Catalana de Sociologia, 11: 191-196.

Wasserman, S. y Faust, K. (1994). Social Network Analysis. Cambridge: Cambridge University Press.

Wengraf, T. (2000). "Uncovering the general from within the particular: from contingencies to typologies in the understanding of cases", en Prue Chamberlayne, J oanna Bornat y Tom Wengraf (ed.): The Turn to Biographical Methods in Social Science. Comparative Issues and Examples. Londres: Routledge, pp. 140-164.

Wengraf, T. (2001). Qualitative Research Interviewing. Biographic Narrative and Semi-Structured Methods. Londres: Sage.

Wheeler, M. A. (2000). "Episodic Memory and Autonoetic Awareness", en Endel Tulving y Fergus I. M. Craik (ed.): The Oxford Handbook of Memory. Oxford/New York: Oxford University Press. 